\title{
LA COMUNICACIÓN DE LA RESPONSABILIDAD SOCIAL CORPORATIVA EN EL ETIQUETADO DE LOS PRODUCTOS
}

\author{
Oscar Juanatey Boga ${ }^{1}$ \\ Valentín Alejandro Martínez Fernández ${ }^{2}$ \\ Universidad de A Coruña \\ María José da Silva Faria ${ }^{3}$ \\ Instituto Superior da Maia - ISMAI. \\ valejand@udc.es \\ oscarib@udc.es \\ mfaria@docentes.ismai.pt
}

https://doi.org/10.17979/redma.2010.01.04.4710

\section{RESUMEN}

La importancia de la transmisión de información de responsabilidad social ha experimentado un notable crecimiento en los últimos años. De este modo, los canales y herramientas de comunicación utilizados son de diversa índole: de nivel social, económico, interno o externo de las organizaciones y otras formas como el etiquetado y rotulado. Así este artículo pretende traducir algunas de las formas más utilizadas por las empresas para la comunicación de la información de responsabilidad social, con especial atención al caso portugués. La metodología empleada fue de un análisis exploratorio de la literatura sobre el tema de este articulo.

Palabras clave: responsabilidad social, comunicación, etiquetado, símbolos.

\footnotetext{
${ }^{1}$ Profesor Contratado Doctor en el Área de Comercialización e Investigación de Mercados de la Universidad de A Coruña. Doctor en Ciencias Económicas y Empresariales por la Universidad de A Coruña. Master MBA en Administración y Dirección de Empresas por la Universidad de A Coruña. Master en Comunicación Empresarial por la Universidad de A Coruña. Licenciado en Ciencias Económicas y Empresariales por la Universidad de A Coruña. Socio Fundador de AEMARK y FISEC

${ }^{2}$ Profesor Titular del Área de Comercialización e Investigación de Mercados de la Universidad de A Coruña. Doctor en Ciencias de la Información por la Universidad Complutense de Madrid. Licenciado en Ciencias de la Información por la Universidad Complutense de Madrid. Master MBA en Administración y Dirección de Empresas por la Universidad de A Coruña. Socio Fundador de AEMARK y FISEC

3 Licenciada em contabilidade pelo ISMAI, 2002. Pós graduação em ciências empresariais pela FEP - Universidade do Porto, 2004. Mestre em ciências empresariais - especialização em contabilidade pela FEP - Universidade do Porto, 2008

Membro da Câmara dos Técnicos Oficiais de Contas, 2001. Membro da corrente cientifica brasileira do neopatrimonialismo, 2006. Docente do ensino superior universitário, 2004. Formadora certificada pelo IEFP, 2004. Contabilista e técnica oficial de contas, 2001. Coordenadora e criadora do CET de
contabilidade e empreendedorismo organizacional no ISMAI
}

\author{
REDMARKA UIMA-Universidad de A Coruña - CIECID \\ Año 2, Número 4, (2010), pp. 95- 104 \\ http://www.redmarka.org/ \\ ISSN 1852-2300
}




\section{Abstract:}

COMMUNICATION OF SOCIAL CORPORATE RESPONSIBILITY IN PRODUCT LABELS

The importance of transmission of social responsibility has grown in recent years. The reports used are different: social, economic, internal or external reports organizations and other forms as the information products. So this article aims to translate some of the most used by companies for the reporting of information of social responsibility particularly in the case of Portugal. The methodology used was an exploratory analysis by defining the main discussions of the topic rigidly the article.

Keywords: social responsibility, communication, labels, symbols. 


\section{Introducción:}

El establecimiento de la Tercera Revolución Industrial en el mundo capitalista, de la informática y las comunicaciones, coincide con la divulgación de la ética empresarial, desarrollo sostenible y responsabilidad social corporativa (Cuevas et al., 2006). derrumbe del socialismo, el desarrollo del ejercicio de una nueva soberanía a nivel mundial (Hardt y Negri 2000), la Tercera Revolución Industrial y la constitución de la sociedad en red (Castells 2009) o la sociedad del conocimiento (Drucker 2000) pueden citarse como los hechos más notables. "Esos factores han puesto al capitalismo, la empresa y el ejercicio del poder capitalista en un lugar hegemónico sin comparación alguna en su historia" (Cuevas, 2009).

De este modo, tal como señala Cuevas (2009), las empresas asumen una mayor presión, pues, a la dimensión económica de la empresa se integra la del cuidado del medio ambiente y la social. Dentro de esta última, el cumplimiento de los derechos universales del hombre y la justicia social ocupan el primer plano. En un segundo nivel se encuentra la educación, la cultura y el deporte. La RSC en su implementación revela prácticas distintas ligadas al desarrollo sostenible: 1) etiquetas ecológicas, comercio justo y marketing ético o responsable, 2) la Inversión Socialmente Responsable; y 3) la evaluación de las políticas de RSC.

En lo que se refiere a las etiquetas ecológicas se puede decir que los consumidores son cada vez más exigentes para con los productos que consumen. La reglamentación en el ámbito de la rotulación de productos comienza a merecer la atención del legislador (Kazazian, 2005). Los programas de etiquetado no son recientes, ya que datan de 1894 en los EEUU con la creación de una empresa que verifica la información concedida por los fabricantes sobre los productos fabricados.

Después de 1940, los primeros rótulos de carácter obligatorio se aplican inicialmente a los productos tóxicos como fertilizantes y pesticidas, y paulatinamente fue ampliado a otros productos. Las primeras recomendaciones en los productos incidían en el ámbito de los cuidados de almacenamiento y de su utilización. Solamente en la década de 1970, la rotulación pasaría a ser un elemento voluntario y no un imperativo legal. En 1977, en Alemania, a través de la iniciativa del Gobierno, fue creado un programa oficial de etiquetado de productos pues se $_{93}$ 
percibió que los rótulos y la información agradaban al consumidor. Así nació el sello Blue Angel. A éste le siguieron el ecologic choice canadiense en 1988, el ecomark japonés de 1989, el green gross y el green seal, ambos norteamericanos en 1990. En 1990, nació también el selo verde brasileño. En la década de 1990 las empresas y los consumidores se mostraron cada vez más exigentes en relación con la información producida y divulgada a los consumidores. El etiquetado pasó a ser mucho más valorado en el área de productos alimentarios y de consumo continuado como la ropa y el calzado, y en momentos recientes ha pasado a serlo en relación con la tecnología y los electrodomésticos.

\section{La importancia del etiquetado de los productos}

Según Harrington y Knight (2001), la rotulación es un atestado en forma se símbolos y grafismos además de literatura como boletines, publicidad, etc. Por lo tanto no deberá ser hecha de cualquier manera y de forma diferenciada entre productos del mismo sector. La armonización de la información se desarrolló a través de la normalización internacional ISO con el objetivo de: Fortalecer les informaciones claras y precisas a los consumidores de manera a que tomen conciencia de sus decisiones; Mejorar el desempeño ambiental y de información en las industrias; y, Garantizar espacio en el mercado, entre otras.

Hoy en día verificamos que el etiquetado de productos, especialmente en lo que se refiere al ámbito ambiental, puede constituir una ventaja competitiva y una forma estratégica de actuación en el mundo de los negocios. Permite agregar valor a la marca y diferenciación con relación a los competidores. Actualmente, son habituales prácticas en las que la rotulación y campañas publicitarias inducen al consumidor a la adquisición de un producto verde sin que éste necesariamente lo sea. Por ejemplo en un anuncio de Pedras Salgadas con la utilización de animales que transportan una botella de agua y el ser humano que asegura la botella caminando por un corredor verde de césped envuelto en flores y árboles que nos producen la sensación de que estamos en el medio natural sacando agua de una fuente de pureza inimaginable, ese agua que sale directamente de su fuente de origen nos hace creer en la naturalidad biológica del producto. En este eslogan, el poder de la ${ }_{94}$ 
naturaleza es infinito y patentiza que el agua gasificada es cien por ciento natural. Le toca al consumidor percibir de la marca Pedras Salgadas no es un producto verde, es decir, no es completamente natural cuando en verdad se nos hace creer que sí lo es.

Otro ejemplo es el del Renault Clio Eco2. Este coche, según la marca, continúa siendo un coche económico con bajo nivel de contaminación, de emisiones sonoras y de cilindrada reducida que utiliza biocombustibles (algunos modelos como el Mégane bioetanol E85, Trafic y el Master biodiesel B30) en que el 5\% de su composición puede servir para hacer plástico reciclado para preservar la naturaleza. La marca pretende ampliar su fabricación, utilización y final de vida cumpliendo la certificación ISO 140001. No obstante, no es por el hecho de que cumplas las normas ISO que la Renault produce un producto verde o ecológico. La reducción de la emisión de la contaminación acústica y del aire puede atenuar los efectos negativos sobre el ambiente, pero no los suple y eso la Renault no lo pone en evidencia en sus campañas de marketing.

Con el 30\% de la cuota del mercado de pinturas en Portugal, la CIN actualmente utiliza en su campaña publicitaria elementos de la naturaleza como animales, playa, nubes, etc. Estos elementos están pintados con colores diversos y diferentes a los que naturalmente existen en la naturaleza. Estos colores más llamativos que son utilizados en la campaña pretenden llamar la atención y crear en el consumidor la percepción e ilusión óptica de un mundo colorido de acuerdo con nuestros gustos personales, la imaginación combinada con la creatividad de los consumidores. Es precisamente esto lo que se pretende con el eslogan de la marca que dice: transformar, crear, CIN me apetece. Las tintas son referidas como ecológicas y biológicas mostrando que no provocan daños al medio ambiente. Observando atentamente el rótulo de los productos comprobamos la utilización de disolventes. Éstos no son nada saludables cuando se utilizan en hospitales, escuelas, oficinas, viviendas, etc. Existen en cantidad reducida sí, pero el hecho es que existen y continúan contaminando el medio ambiente.

En estos casos (Pedras Salgadas, Renault Clío y CIN) están latentes situaciones que retratan la realidad del marketing engañoso. El consumidor debe estar alerta 
para descodificar los mensajes que existen por detrás de los anuncios publicitarios. Hay marcas que de forma natural llaman la atención sobre un marketing verde sin engaños. Un ejemplo es el de la Compal. La marca comenzó con la comercialización de pulpa de tomate y a lo largo del tiempo ha ido ampliando su gama de productos a los zumos y néctares. La marca llama la atención sobre la utilización de materias primas naturales sin utilización de colorantes ni conservantes con una presentación de un producto diferente y con calidad. La Agros Biológica es otro ejemplo de producto que se pauta por la naturalidad, por la ecología y el ámbito ecológico de un producto verde. Con el objetivo de preservar la salud y el bienestar, la línea de productos de la Agros Biológica creó una red de producción de leche y transformación de sus derivados de forma natural. Esta estrategia de competencia está, de forma semejante, presente en otras marcas que también se guían por la naturalidad de los productos verdes y por su éxito y crecimiento tanto en el espacio del mercado portugués como en el del extranjero.

Observando dos productos, un verde y otro no verde, fácilmente el consumidor se apercibe de la diferencia entre ambos a través de los símbolos que encuentra en el rótulo de cada envase.

Cada símbolo tiene un significado y no todos ellos son conocidos por sus consumidores. La falta de información lleva los consumidores, a adquirir productos de los cuales no se conoce verdaderamente los riesgos e implicaciones para la salud y para el medio ambiente. De dicha falta de información se aprovechan algunas marcas de productos para influenciar al consumidor en el acto de compra. La buena información lleva al consumidor a distinguir los buenos de los malos símbolos y los buenos de los malos productos.

\section{Información de los símbolos en el etiquetado de los productos}

Un conjunto de símbolos nos permite hoy efectuar esa distinción. Existen los que declaran una certificación de calidad y que cumplen los requisitos y las normas legales en vigor. Un ejemplo es el símbolo APCER, que puede tener diferentes formatos, colores e inscripciones. A pesar de ello, el producto continúa teniendo 
certificación al igual que ocurre con otras certificaciones como es el caso de la SGS y la CERTIF. La certificación de los productos es una garantía para el consumidor. El caso de la invasión de los productos procedentes de China, India y del norte de África, que son una gran amenaza para el comercio europeo y para reducción de calidad para el consumidor, llevó a la creación de la diferenciación de productos. La entrada de productos provenientes del exterior para Portugal tiene que venir acompañada de la certificación respectiva. En todos los rótulos de los productos en que se encuentre inscripción $C E$, los consumidores saben que la procedencia de este producto es originaria de un país de la Unión Europea, y esto garantiza su adecuación a las directivas europeas.

La certificación de calidad obliga a que una empresa implemente un sistema de gestión de calidad. Ese sistema deberá obedecer a la normativa en vigor ISO y/o NP EN. Las normas ISO certifican una diversidad de productos y aspectos, excepto productos de electrónica y de electricidad. Además de la certificación ISO, inscrita en los rótulos de los embalajes de los productos, otros símbolos pueden ser encontrados, pero no todos se refieren a la certificación de calidad. El símbolo del punto verde, en el rótulo no es sinónimo de que el mismo esté certificado y presente relaciones con un producto verde o biológico, sólo por el hecho mismo de que el color sea verde. Este símbolo no implica que el producto sea reciclable, sino que cumple la legislación en el ámbito de la gestión de residuos de embalajes. Asociado al punto verde hay un conjunto vasto de símbolos que se refieren al tratamiento de residuos tales como el eco puntos (amarillo, azul y verde) y el eco puntos con contenedores de recogida selectiva de pilas y aparatos electrodomésticos. Adicionalmente, en las farmacias de Portugal se puede encontrar un pequeño recipiente para depositar los medicamentos fuera de plazo. Tanto los depósitos de contaminantes como la rotulación ambiental de productos es, en este momento, un elemento de orden público y privado.

Los rótulos verdaderamente ecológicos nacieron por el reglamento europeo de 1992 revisado en 2000 (Reglamento (CE) no 1980/2000 (JOCE L 237 de 21.9.00)) que garantizaba que los productos tienen un impacto muy reducido sobre el nivel ambiental durante su ciclo de vida. Este símbolo atesta que el producto es responsable con el medioambiente y es totalmente ecológico. Este rótulo no es 
aplicable a bebidas, productos alimentarios y farmacéuticos ni a productos con sustancias peligrosas. Para que un producto tenga el rótulo ecológico, la empresa de fabricación tiene que cumplir con un conjunto de criterios definidos en el boletín diario de las Comunidades (eco-label). En Portugal, la atribución del rótulo ecológico es conferida por la Dirección General de Actividades Económicas.

Los rótulos de los productos pueden ser agrupados en tres grandes tipos: rótulos de tema único, rótulos negativos y ecolabels. Los rótulos de tema único informan sobre características de los productos, por ejemplo, performance, emisiones, consumo, etc. Los rótulos negativos evidencian información que alerta sobre la peligrosidad de los productos en causa. Los ecolabels informan sobre el análisis del ciclo de vida del producto y de sus consecuencias ambientales. Además de los ecolabels, otras iniciativas fuera de la Unión Europea fueron y son emprendidas para garantizar la rotulación ecológica.

\section{Iniciativas de rotulación en el mundo}

El primer rótulo ambiental desarrollado en el mundo fue el de Blue Angel, implementado en Alemania (1977). Este programa fue una innovación y su recepción entre los consumidores y las empresas fue bien considerada y proporcionó todo su empeño para mejorar en desempeño ambiental. El rótulo Blue angel es administrado por tres organizaciones: la Agencia federal del medio ambiente, que define los criterios para la confección del rótulo y conduce a testes de impacto ambiental en todo el ciclo de vida de los productos; la Comisión que delibera sobre el sello ambiental está constituida por representantes de la industria de grupos ambientales, por representantes de sindicatos y de la comunidad científica. Tiene la atribución de las decisiones finales sobre categorías de productos y criterios para la concesión del sello; y, el Instituto Alemán de Calidad y Rotulación discute los criterios para las categorías de los productos, coordina la evaluación de un producto y la negociación de un contrato con los productores para la utilización del rótulo.

El programa se disecciona por directrices: el rótulo sólo puede ser utilizado para productos; los productos deben estar destinados al consumidor final; el mercado 
debe ser lo suficientemente amplio que permita la competencia; los criterios estipulan limitación del número de rótulos autorizados, y los criterios son válidos por un periodo indeterminado siendo revistos periódicamente. Para que los productos pasen a tener en su rótulo este sello, deben ser sometidos a análisis por el Instituto alemán de garantía de calidad y rotulación. Para ciertos grupos de productos, el Instituto exige testes de peritos independientes, en general para la consumen quality test organización. Los fabricantes extranjeros pueden solicitar la concesión del rótulo para sus productos, simplemente basta con que sigan los procedimientos exigibles por los productores alemanes (Corrêa, 1998).

Los primeros rótulos fueron conferidos en 1979 para 48 productos; en 1995, para 4.353 productos; en 1998, para 4.135 y en 2003, para 3.700. El Blue Angel es un rótulo bastante conocido por los consumidores alemanes. Los criterios desarrollados por el programa son cada vez más utilizados por el Estado, aunque la legislación alemana no obligue a incluir rotulación entre las especificaciones para las compras gubernamentales. En Alemania como miembro de la Unión Europea coexisten otros sellos con el Blue Angel, contribuyendo siempre a generar productos menos perjudiciales para el medio ambiente.

Canadá fue el segundo país que desarrolló un programa de este tipo en la década de 1980 y que denominó Environmental choice program (ECP). Inicialmente, fue implementado y observado por el Gobierno y, posteriormente, fue utilizado y controlado por TerraChoice Environemental Services Inc.

El rótulo es conocido como EcoLogo, y su símbolo está constituido por tres palomas ligadas bajo la forma de una hoja de maple estilizada. En marzo de 1990 fueron conferidos los primeros sellos ecólogos. En 1995, fueron conferidos sellos a 1.500 productos. El programa sigue cinco principios para la determinación de las categorías de productos y criterios técnicos: estimular el uso eficiente de los recursos renovables; facilitar la reducción, reutilización y reciclaje de contaminantes a nivel industrial, comercial o de consumo; contribuir para la protección de los ecosistemas y la diversidad de las especies, y estimular el uso apropiado de componentes químicos en los productos. Tal como ocurre con el Blue Angel, este sello conferido en los rótulos no incluye explicación para su atribución. Los 
criterios son revistos periódicamente y los tests y auditorías ambientales verificadas cada seis meses como máximo. La empresa fija bajo de la observación regular de TerraChoice. El programa canadiense acepta la certificación de instituciones internacionalmente reconocidas. Estos dos programas sirven de referencia a otros programas ambientales implementados en Asia, EEUU y Europa.

El programa de rotulación ambiental del Japón conocido como EcoMark fue creado en 1989 por la Japonese Environmental Agency del Ministerio del Medio Ambiente bajo observación de la Japonese Environmental Association (JEA). El rótulo presenta dos brazos que abrazan el globo, representando el deseo de proteger a la Tierra con sus propias manos que forman la letra $E$ de environmental, earth y ecology. En la parte superior está la expresión friendly to the Herat en japonés (Corrêa, 1998).

Los criterios de este programa consideran el ciclo de vida de los productos, siendo tenidos en cuenta también factores como la seguridad y la calidad. La empresa que utiliza este rótulo debe adoptar medidas de combate a la contaminación durante el proceso de producción; el uso del producto deberá ser eficiente en la conservación de la energía; la calidad y la seguridad del producto deben obedecer a la legislación en vigor manteniendo el producto en un precio competitivo. En 1989, en el momento de lanzamiento del programa, los rótulos fueron conferidos a 46 productos y en 1995, a 2.322.

Según Corrêa (1998), el programa de rotulación ambiental de los EEUU fue creado en 1989 por una organización no gubernamental - Greenseal -, sin fines lucrativos, con el objetivo de establecer patrones de rotulación ambiental de productos para orientar al consumidor en la compra de aquellos que causan menores daños al medio ambiente. Como símbolo presenta un globo encubierto por una senal de verificación veree - checmark. El programa define como objetivos principales el establecimiento de patrones y criterios para reducir la contaminación química tóxica, el consumo de energía, los impactos en los recursos acuáticos, la vida salvaje, la atmósfera, el calentamiento global y por añadidura que los productos estén de acuerdo con patrones mínimos de desempeño. En el proceso de definición de categorías de productos y servicios son aceptadas propuestas, tanto si 
procedentes de la industria como de cualquier ciudadano, de nuevas categorías a ser incluidas en los estudios. Para asesorar el proceso de formulación de criterios son creados, cuando se asemeja necesario, grupos especiales. Las sugestiones son evaluadas $y$, si se consideran pertinentes, son incorporadas. Además de los criterios establecidos, el producto en cuestión debe también cumplir con todas las normas de seguridad y desempeño así como todas las exigencias ambientales legales.

Los criterios son revistos cada tres años para acompañar los avances tecnológicos y estimular el mejoramiento ambiental. Para que las empresas reciban la autorización para utilización del rótulo, éstas deben proporcionar informaciones sobre métodos y procesos de producción además de someter sus productos a testes.

El programa de rotulación ambiental de los países nórdicos, el Nordic Swan fue adoptado en 1989 por el consejo nórdico de ministros donde están representadas Dinamarca, Finlandia, Islandia, Noruega y Suecia. El rótulo se basa en el símbolo del consejo nórdico y presenta un cisne blanco sobre un fondo verde. La expresión rótulo ambiental figura en la parte superior en el idioma del país y en la parte de abajo se encuentra el atributo ambiental correspondiente. La definición de criterios tiene en cuenta el análisis del ciclo de vida del producto, pero seleccionando solamente los impactos ambientales más importantes. Los criterios comprenden procedimientos de verificación basados en métodos internacionales fijados de modo a maximizar la compatibilidad con otros programas. En 1991 fueron conferidos los primeros rótulos. En este momento, Suecia tiene también otro programa de rotulación administrado por un grupo ambiental. Las empresas suecas, que ya disponían de su propio sello, se muestran hoy poco receptivas a adquirir el rótulo Nordic Swan.

El programa de rotulación de Singapur, el Greenlabel, fue lanzado en 1992 con la finalidad de prestar informaciones a los consumidores y asegurar su contabilidad a los esquemas de rotulación ambiental implementados en los países industrializados. Tiene como marca una hoja verde rodeada por la expresión GreenLabel Singapore (Corrêa, 1998). El programa es administrado por el Ministerio de Medio Ambiente y comprende un comité consultivo integrado por representantes de sectores 
empresariales, de grupos ambientales y de medios académicos. Tiene como objetivos estimular a los consumidores a que adquieran productos de menor daño al medio ambiente y paralelamente incentivar a los productores a que consideren la variable ambiente en sus estrategias empresariales. La definición de criterios se basa en el análisis del ciclo de vida y en el impacto que el producto ejerce sobre el medio ambiente.

Los programas de rotulación ambiental significan hoy un adelanto en la ética y en la supervivencia humana a nivel de la responsabilidad social. La rotulación funciona como señal de reconocimiento de las prácticas correctas para el fabricante como diferencial competitivo en relación con sus competidores. La conciencia para la práctica de acciones socialmente responsables y la divulgación de información en los rótulos de información que interesa al buen uso de los productos fornece a los consumidores un manantial de producción escrita que valoriza a la empresa fabricante.

\section{Conclusión}

En síntesis, podemos afirmar que la responsabilidad social es usada estratégicamente para fines organizacionales y, en muchos casos, esos fines son incluso coincidentes con las ayudas y demás auxilios a la sociedad o comunidad en que las compañías desarrollan su actividad. La comunicación de estas estrategias y de las prácticas de responsabilidad social es en su gran mayoría hecha a través de los mass media o de campañas de marketing social.

En todo caso, en los últimos años ha alcanzado gran relevancia la utilización de estrategias de comunicación a través del etiquetado y rotulado de productos, para hacer llegar una información más clara y transparente al consumidor final. En este sentido la implementación de sellos de garantía y otros tipos de rotulado relativos a la preocupación y respeto ambiental por parte de las empresas comercializadoras, ha tenido un incesante crecimiento que pone de relieve el cada vez más relevante peso de las prácticas de responsabilidad social corporativa en todos sus ámbitos de 
aplicación, y en concreto en lo que se refiere al contexto medioambiental.

A pesar, en todo caso, de que podemos considerar elevado el retorno social de una empresa que practica la responsabilidad social, ha de considerarse que tal y como señala Guedes (2000) no se puede disponer de la información de todas las variables sobre las que impacta este tipo de prácticas tales como, tal y como sugiere esta autora, la imagen de ventas; la marca del producto; la valorización de la empresa en el mercado; el retorno publicitario; la productividad del capital humano al servicio de la empresa; la motivación y empeño de los empleados; y, las ganancias sociales por los cambios de comportamiento en la sociedades.

\section{Bibliografía}

CASTELLS, Manuel (2009) The Rise of the Network Society: Information Age: Economy, Society, and Culture (Information Age Series), vol. 1, WileyBlackwell, $2^{\mathrm{a}}$ edition with a New Preface edition.

CORRÊA, Leonilda (1998) BCGD - Comércio e Meio Ambiente: atuação diplomática brasileira em relação ao selo verde, Brasília, Instituto Rio Branco.

CUEVAS MORENO, R. y GARZA ARROYO, A. (2006) "La relación entre la Responsabilidad Social de la Empresa y la Ética de los Negocios: Una solución a partir de la dialéctica materialista", CLADEA, 41 st Annual Assembly Latin America \& European Union. Opportunities and Challenges, Montpellier Francia, 10, 11, 12 y 13 de Septiembre.

CUEVAS MORENO, Ricardo (2009) Ética y responsabilidad social de la empresa: su concepción del hombre. Polis, Santiago, vol. 8, nº. 23.

DRUKER, Peter (2000) "O futuro já chegou", in Exame Digital, São Paulo, abril, ed. 710. Ano 34. N. 6. mar., pp. 113-126.

GUEDES, Rita de Cássia (2000) Responsabilidade social e cidadania 
empresariais: conceitos estratégicos para as empresas face à globalização. Disertación (Máster en Administración de Empresas de la Pontificia Universidad Católica de São Paulo). São Paulo, PUC/SP.

HARDT, M et NEGRI, A. (2000) Empire, Exils Editeur, traduit de Paméricain par Canal, D-A. Paris.

HARRINGTON, H.J.; KNIGHT, A. (2001) A implementação da ISO 14000: como actualizar o SGA com eficácia, São Paulo, Atlas.

KAZAZIAN, T. (2005) Design e desenvolvimento sustentável: Haverá a idade das coisas leves, São Paulo, Editora Senac.

SCHMIDHEINY, S.; HOLIDAY JR, C.; WATTS, P. (2002) Cumprindo o prometido: casos de sucesso de desenvolvimento sustentável, Rio de Janeiro, Editora Campus.

\section{Para citar este artículo:}

Juanatey Boga, Oscar - Martínez Fernández, Valentín Alejandro - da Silva Faria, María José (29-042010). LA COMUNICACIÓN DE LA RESPONSABILIDAD SOCIAL CORPORATIVA EN EL ETIQUETADO DE LOS PRODUCTOS.

REDMARKA - CIECID - Unidad de Investigaci $\tilde{A}^{3} n$ en Marketing Aplicado-Universidad de A CoruÃ $\pm a$, Número 4, V1, pp.95-104

ISSN 1852-2300

URL del Documento : cienciared.com.ar/ra/doc.php?n=1313

URL de la Revista : cienciared.com.ar/ra/revista.php?wid=39 